\title{
Aluminum-lithium clusters: First-principles simulation of geometries and electronic properties
}

\author{
Jaakko Akola* and Matti Manninen \\ Department of Physics, University of Jyväskylä, FIN-40351 Jyväskylä, Finland
}

(Received 3 January 2002; published 24 June 2002)

\begin{abstract}
The geometries and electronic properties of small lithium-rich $\mathrm{Al}_{N} \mathrm{Li}_{5 N}(N=1-6,10)$ clusters are studied using first-principles simulations. Aluminum ions form a compact inner core configuration in the clusters that changes into a chainlike skeleton embedded in a lithium surrounding as the cluster size increases. This behavior restricts $s-p$ hybridization effects and causes separate $s$ and $p$ bands in the electronic energy spectrum. A significant charge transfer from $\mathrm{Li}$ ions and nearby $\mathrm{Al}$ ions strengthens ionic $\mathrm{Al}-\mathrm{Li}$ bonds, while $\mathrm{Al}-\mathrm{Al}$ bonds gain a more covalent nature. The evolution of some bulk properties of $B 2$ and $B 32$ phases of AlLi alloys is studied by constructing 59- and 145-atom $\mathrm{Al}_{N} \mathrm{Li}_{M}(N \approx M)$ clusters based on a truncated rhombic dodecahedron. Tetrahedrally coordinated covalent $\mathrm{Al}-\mathrm{Al}$ bonds of $B 32$ clusters affect the electronic properties and hardness.
\end{abstract}

DOI: 10.1103/PhysRevB.65.245424

PACS number(s): 36.40.Cg, 36.40.Mr, 73.22.-f

\section{INTRODUCTION}

Atomic clusters are intermediate objects between a single atom and infinite bulk matter and provide an interesting laboratory to study how the bulk properties emerge with increasing system size. Furthermore, they offer a possibility to investigate different size-evolutionary patterns of different materials. For example, the development of properties such as cluster geometries, thermodynamic stability, abundance distributions, electronic and vibrational spectra, dissociation and ionization energies, and chemical reactivity depends on the material in question.

A major breakthrough in metal cluster research was the observation of the electronic shell effects of homonuclear alkali-metal clusters $(\mathrm{Na}, \mathrm{K})$ in the experimental mass abundance spectrum. ${ }^{1}$ In these studies, the most abundant cluster sizes (i.e., shell closings) were found to match the prediction of the spherical jellium model (SJM), where the ionic background density is uniform. ${ }^{2,3}$ The electronic shell structure found in other simple and noble-metal clusters ${ }^{4}$ induced a rapid growth of interest in homonuclear metal clusters. Bimetallic clusters and their possible connection to the SJM also attracted interest, and many studies of such clusters were performed, with the results being interpreted as favoring SJM effects or otherwise, depending on the composition of the system. ${ }^{5-7}$ An example of recent work is the experimental study by Heinebrodt et al. ${ }^{7}$ on $\mathrm{Au}_{N} X_{M}{ }^{+}(X=\mathrm{Al}, \mathrm{In}, \mathrm{Cs})$ clusters, where each mixture displays distinct properties regarding charge transfer and SJM effects.

Both pure $\mathrm{Al}$ and $\mathrm{Li}$ clusters have been studied extensively using the SJM ${ }^{8-14}$ and the measurements follow SJM predictions relatively well in small clusters. There are, however, some properties [e.g., high ionization potential (IP) of small Al clusters ${ }^{14,15}$ and ionic geometries of small Li clusters $(N \leqslant 20)$ (Ref. 12)] that differ from other alkali metals. The underlying reasons for these phenomena are the relatively low-lying unoccupied $2 p$ shell of $\mathrm{Li}$ and the uppermost $3 p$ electron of $\mathrm{Al}$ atoms. Similarly, mixtures of these elements exhibit properties ${ }^{16-19}$ involving charge transfer from $\mathrm{Li}$ ions to $\mathrm{Al}$ orbitals and $\mathrm{Al}-\mathrm{Al}$ bonds. This was recently demonstrated by $\operatorname{Kumar}^{19}$ for a closed-shell ("magic") decahedral $\mathrm{Al}_{10} \mathrm{Li}_{8}$ cluster (38 valence electrons), where ionic $\mathrm{Al}-\mathrm{Li}$ and covalent $\mathrm{Al}-\mathrm{Al}$ bonds could be identified.

We report here our findings for lithium-rich $\mathrm{Al}_{N} \mathrm{Li}_{5 N}(N$ $=1-6,10)$ cluster alloys and $\mathrm{Al}_{N} \mathrm{Li}_{M}(N \approx M)$ cluster analogies for $B 2$ and $B 32$ phases of bulk AlLi, motivated in part by the possibility of building larger cluster-assembled entities from $\mathrm{AlLi}_{5}$ clusters. This suggestion came initially from Cheng et al., ${ }^{16}$ who observed that $\mathrm{AlLi}_{5}$ exhibits an atomiclike electronic structure, where the valence electron density is localized around $\mathrm{Al}$ atoms giving the cluster a similar character as to rare-gas atoms. ${ }^{16}$ We explore in detail some ideas of coating $\mathrm{Al}$ clusters by lithium, ${ }^{18}$ and we investigate whether it is possible to assign the bulk properties of AlLi even at relatively small cluster sizes. The bulk AlLi alloy has been observed to possess remarkably high hardness despite its low density, and the AlLi phase diagram ${ }^{20}$ shows that its melting point $(T \sim 970 \mathrm{~K})$ is even higher than that of pure aluminum $(T \sim 930 \mathrm{~K})$. These properties are related to the B32 (Zintl) phase of AlLi, where bcc unit cells are tetrahedrally occupied by $\mathrm{Al}$ and $\mathrm{Li}$ atoms, respectively. As shown by Guo et al., ${ }^{21}$ a remarkable charge transfer from $\mathrm{Li}$ atoms to covalent $\mathrm{Al}-\mathrm{Al}$ bonds and the underlying diamond sublattice cause the $B 32$ alloy to exhibit diamondlike properties. We also study the metastable $B 2(\mathrm{CsCl})$ phase of $\mathrm{AlLi}$, which has been predicted theoretically to exist at high pressure. $^{21}$

The first-principles Born-Oppenheimer local-spin-density molecular-dynamics (BO-LSD-MD) simulation method and the cluster generation processes are described briefly in Sec. II. The results for $\mathrm{Al}_{N} \mathrm{Li}_{5 N}(N=1-6,10)$ and $\mathrm{Al}_{N} \mathrm{Li}_{M}(N$ $\approx M$ ) cluster alloys are presented in Sec. III, and we summarize our findings in Sec. IV.

\section{METHOD}

The calculations are performed using the BO-LSD-MD method $^{22}$ employing the density functional theory (DFT) based Kohn-Sham (KS) formalism in conjunction with separable nonlocal, norm-conserving pseudopotentials for the $3 s^{2} 3 p$ and $2 s$ valence electrons of aluminum and lithium, 

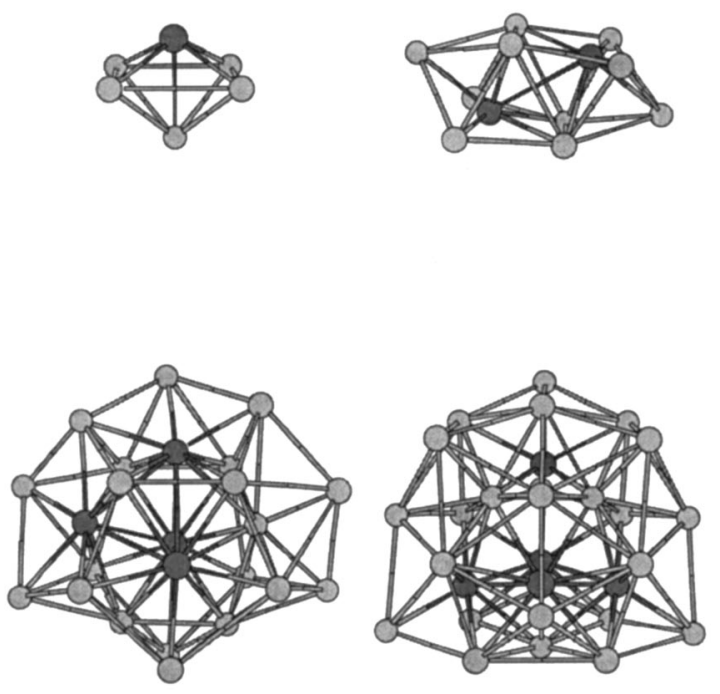
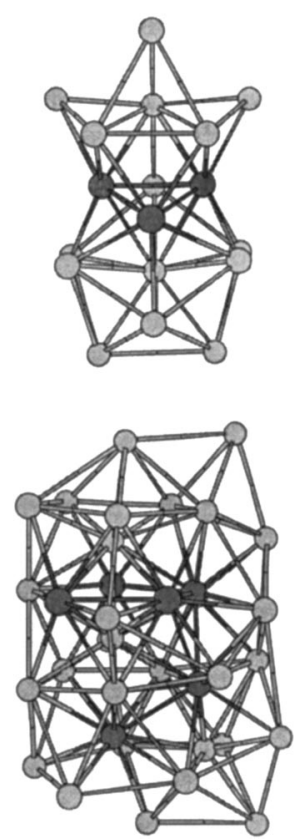

FIG. 1. Lowest-energy structures of $\mathrm{Al}_{N} \mathrm{Li}_{5 N}$ $(N=1-6)$ clusters. $\mathrm{Al}$ atoms are black; $\mathrm{Li}$ atoms are gray. respectively. $^{23}$ We use two parametrizations for the exchange-correlation functional of the total energy: the LSD approximation of Vosko, Wilk, and Nusair ${ }^{25}$ (VWN) and the generalized gradient approximation (GGA) of Perdew, Burke, and Ernzerhof ${ }^{24}$ (PBE). Ions move according to classical molecular dynamics, with the electronic HellmannFeynman forces evaluated at each time step from the valence electron density, and the method does not use supercell techniques in calculating the total energy of a finite system; i.e., there are no repeated images of the system. This makes it possible to calculate charged systems and systems with large multipole moments accurately and efficiently, and IP's and detachment energies (DE's) can now be obtained as total energy differences between neutral and charged clusters.

Optimized $\mathrm{Al}_{N} \mathrm{Li}_{5 N}$ cluster geometries and corresponding energies are determined using a steepest-descent-like minimization (with LSD approximation and GGA, separately) without symmetry constraints. The related starting configurations are generated via $a b$ initio molecular dynamics (time step $\tau=3$ fs) at high temperature $(T=1000-1200 \mathrm{~K})$ combined with a simulated annealing procedure. The high temperature is used to ensure sufficient mobility of Li ions (which move slowly at low temperatures) and shape fluctuations. In order to speed up the simulations the simulated annealing is performed using the LSD approximation instead of the more sophisticated (and expensive) GGA. In addition to the customary annealing procedure, each simulation ends with a slow exponential temperature decay (half-life $\lambda$ $\sim 1$ ps, cooling time $\Delta t=3-4 \mathrm{ps}$ ), where the resulting final structure generally has the lowest total energy among all structures considered. In addition, the isomers are modified further by swapping the positions of $\mathrm{Al}$ and $\mathrm{Li}$ atoms and by changing the positions of outermost $\mathrm{Li}$ atoms.

\section{RESULTS}

\section{A. $\mathbf{A l}_{N} \mathbf{L i}_{5 N}$ clusters}

The lowest-energy structures of $\mathrm{Al}_{N} \mathrm{Li}_{5 N}$ clusters ( $N$ $=1-6)$ are presented in Fig. 1 . AlLi $\mathrm{Li}_{5}$ has the $C_{4 v}$ structure found by Cheng et al. where the $\mathrm{Al}$ atom is capped by $\mathrm{Li}$ atoms, and $\mathrm{Al}_{2} \mathrm{Li}_{10}$ has the $D_{2 d}$ structure comprising two

TABLE I. Properties of $\mathrm{Al}_{N} \mathrm{Li}_{5 N}$ clusters: number of valence electrons, averaged binding energy (LSD/

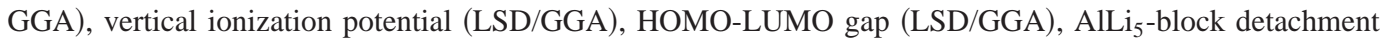
energy (GGA), second difference of total energy (GGA), and Al-Al bond lengths (GGA).

\begin{tabular}{lccccccc}
\hline \hline$N$ & $N_{e l}$ & $\bar{E}_{b}(\mathrm{eV} /$ atom $)$ & $v I P(\mathrm{eV})$ & $E_{g}(\mathrm{eV})$ & $D(\mathrm{eV})$ & $-\Delta^{2} E(\mathrm{eV})$ & $d(\mathrm{Al}-\mathrm{Al})(\AA)$ \\
\hline 1 & 8 & $1.37 / 1.18$ & $5.08 / 4.95$ & $1.20 / 1.32$ & $\ldots$ & $\ldots$ & $\ldots$ \\
2 & 16 & $1.58 / 1.37$ & $4.11 / 3.97$ & $0.68 / 0.77$ & 2.16 & -0.92 & 3.15 \\
3 & 24 & $1.70 / 1.48$ & $4.08 / 3.95$ & $0.57 / 0.60$ & 3.09 & 0.56 & $2.76-2.85$ \\
4 & 32 & $1.74 / 1.51$ & $3.97 / 3.80$ & $0.42 / 0.45$ & 2.52 & -1.20 & $2.76-3.14$ \\
5 & 40 & $1.81 / 1.57$ & $3.95 / 3.80$ & $0.54 / 0.57$ & 3.72 & 0.57 & $2.77-2.93$ \\
6 & 48 & $1.83 / 1.59$ & $3.74 / 3.60$ & $0.22 / 0.23$ & 3.15 & $\ldots$ & $2.77-2.91$ \\
$\ldots$ & $\ldots$ & $\ldots$ & $\ldots$ & $\ldots$ & $\ldots$ & $\ldots$ & $\ldots$ \\
10 & 80 & $1.91 / 1.66$ & $3.56 / 3.48$ & $0.08 / 0.10$ & $\ldots$ & $\ldots$ & $2.76-2.99$ \\
\hline \hline
\end{tabular}



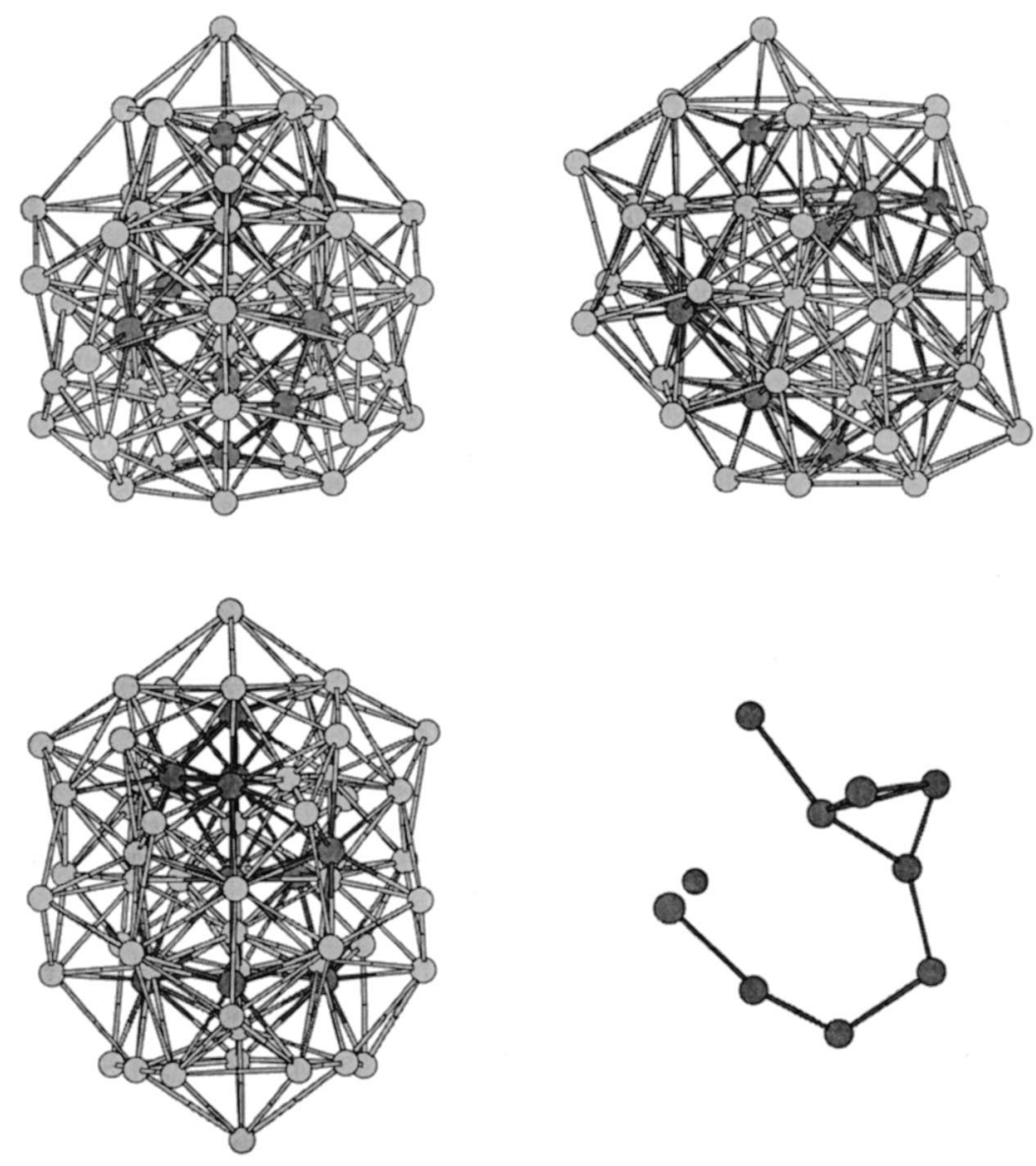

FIG. 2. Lowest-energy structure of $\mathrm{Al}_{10} \mathrm{Li}_{50}$ cluster seen from three different angles (front, right, and top) and its $\mathrm{Al}$ skeleton (right). distorted $\mathrm{AlLi}_{5}$ units suggested by the same authors. The long $\mathrm{Al}$-Al distance $(3.15 \AA$; see Table $\mathrm{I})$ of $\mathrm{Al}_{2} \mathrm{Li}_{10}$ underscores the division into two subunits. The idea of individual $\mathrm{AlLi}_{5}$ blocks cannot be extended to larger cluster sizes, as can be seen in the prolate $C_{s}$ structure of $\mathrm{Al}_{3} \mathrm{Li}_{15}$, where the $\mathrm{Al}$ atoms form an isosceles triangle capped from above and below by two lithium units. The aluminum atoms of $\mathrm{Al}_{4} \mathrm{Li}_{20}$ form a $C_{2 v}$ "butterfly" configuration surrounded by $\mathrm{Li}$ atoms. The cluster is oblate and shows an icosahedral fivefold packing in accordance with the structures of pure mediumsized $\mathrm{Al}$ and $\mathrm{Li}$ clusters $\left(10<N_{A l}<20\right.$ and $\left.N_{L i}>20\right)$. The aluminum "core" of $\mathrm{Al}_{5} \mathrm{Li}_{25}$ has a $C_{s}$ symmetry, where the uppermost $\mathrm{Al}$ atom has only one $\mathrm{Al}$ nearest neighbor. In contrast to $\mathrm{Al}_{4} \mathrm{Li}_{20}$, the packing of the cluster is not icosahedral, but it resembles more the bcc lattice and particularly the $B 2$ phase of AlLi bulk. The mixing of $\mathrm{Al}$ and $\mathrm{Li}$ atoms is also present in $\mathrm{Al}_{6} \mathrm{Li}_{30}$, where the $\mathrm{Al}$ atoms are arranged in a low-coordinated $C_{3 v}$ unit. The overall shape of the cluster is elongated and icosahedral patterns (even a 19-atom double icosahedron) can be identified.

In Fig. 2 the lowest-energy structure of the $\mathrm{Al}_{10} \mathrm{Li}_{50}$ cluster is shown from three perspectives-front, right, and above-and the pure aluminum skeleton of the cluster is shown from the right. As already seen in $\mathrm{Al}_{4} \mathrm{Li}_{20}$ and $\mathrm{Al}_{6} \mathrm{Li}_{30}$, it is easy to assign icosahedral fivefold patterns in the cluster geometry. The mixing of $\mathrm{Al}$ and $\mathrm{Li}$ atoms continues from $\mathrm{Al}_{6} \mathrm{Li}_{30}$, causing the embedded $\mathrm{Al}$ skeleton to adopt a chainlike form with each $\mathrm{Al}$ atom having on average two $\mathrm{Al}$ nearest neighbors. This is similar to the lithium-rich bulk alloy of $\mathrm{Al}_{4} \mathrm{Li}_{9}(B 2 / m)$, where $\mathrm{Al}$ atoms tend to form "zigzag" chains. ${ }^{21}$ Furthermore, it is observed both experimentally and theoretically that other Li rich phases where the Li-content has been increased do not exist, but decompose into the $B 2 / m$ phase of $\mathrm{Al}_{4} \mathrm{Li}_{9}$ and pure lithium. ${ }^{20,21}$

The properties of $\mathrm{Al}_{N} \mathrm{Li}_{5 N}$ clusters are listed in Table I. In order to illustrate the differences between the LSD approximation and GGA we have included both values for average binding energy $\left(\bar{E}_{b}\right)$, vertical ionization potential (vIP), and energy gap $\left(E_{g}\right)$ between the highest occupied and lowest occupied molecular orbit (HOMO-LUMO). The general trends are the following: $\bar{E}_{b}$ increases monotonically whereas the vIP and and $E_{g}$ decrease almost monotonically (note $E_{g}$ of $\mathrm{Al}_{5} \mathrm{Li}_{25}$ ). These properties are characteristic of metal clusters, and linear extrapolations of $\bar{E}_{b}$ and vIP values (GGA) with respect to $N_{e l}^{-1 / 3}$ lead us to theoretical bulk estimates $\left(\bar{E}_{C}=2.09 \mathrm{eV}, W=2.79 \mathrm{eV}\right)$ that are closer to the corresponding values of pure bulk $\mathrm{Li}(1.63 \mathrm{eV}$ and $2.38 \mathrm{eV})$ than $\mathrm{Al}(3.39 \mathrm{eV}$ and $4.25 \mathrm{eV}){ }^{26,27}$ Furthermore, Al-Al bond lengths are distributed over a broad range [for $\mathrm{Al}$ bulk $d(\mathrm{Al}-\mathrm{Al})=2.86 \AA]$, and it is difficult to assign any trends.

Inspection of Table I shows that the effect of the GGA is to lower both $\bar{E}_{b}$ and the vIP, and the HOMO-LUMO gap is increased in all cases. The latter is a typical improvement to the LSD approximation ${ }^{28}$. The bond lengths increase due to the GGA (1\%-3\% for the Al-Al bonds). 


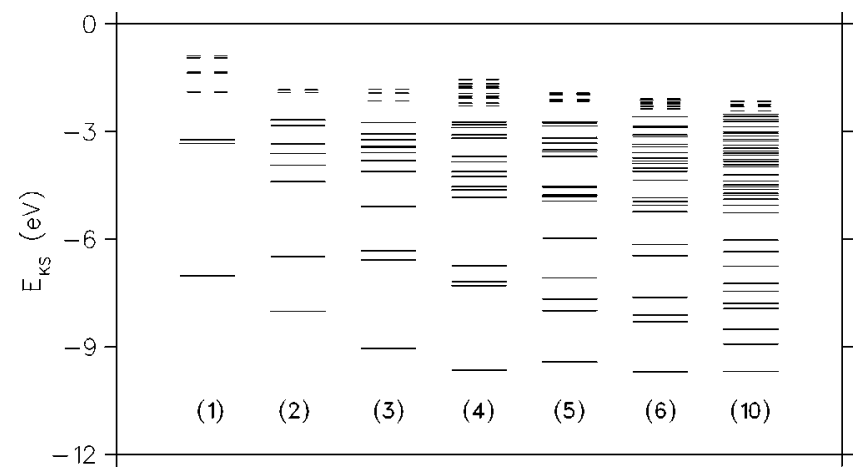

FIG. 3. KS-GGA energy levels of $\mathrm{Al}_{N} \mathrm{Li}_{5 N}(N=1-6,10)$ clusters. The shorter lines correspond to unoccupied orbitals.

Indications of the relative stabilities of different clusters can be obtained from Table I, where the $\mathrm{AlLi}_{5}$-block detachment energies $(D)$ and corresponding second differences of total energy $\left(-\Delta^{2} E\right)$ are also reported. The oscillating patterns in both of these quantities suggest that $\mathrm{Al}_{3} \mathrm{Li}_{15}$ and $\mathrm{Al}_{5} \mathrm{Li}_{25}$ are the most stable clusters, and this is consistent with the vIP and $E_{g}$ values (see also Fig. 3). In spite of the compact geometry of $\mathrm{Al}_{5} \mathrm{Li}_{25}$ (40 electrons), we cannot associate its relatively high stability to the SJM (note also the high degeneracies of electronic levels of $\mathrm{Al}_{5} \mathrm{Li}_{25}$ in Fig. 3). ${ }^{29}$

The KS energy eigenvalues of $\mathrm{Al}_{N} \mathrm{Li}_{5 N}$ clusters calculated with GGA are shown in Fig. 3. As noted by Cheng et al., ${ }^{16}$ we observe that the electronic structure of the $\mathrm{AlLi}_{5}$ unit shows that the charge of $\mathrm{Li}$ atoms moves to the Al-Li bonds while the $3 p$ shell of $\mathrm{Al}$ is filled. This idea can be continued to $\mathrm{Al}_{2} \mathrm{Li}_{10}$, where two $\mathrm{AlLi}_{5}$ units are combined. Investigation of the resulting KS orbitals reveals that they can be considered in terms of ordinary molecular orbitals of an atomic dimer resulting in the sequence $1 \sigma_{g}\left|1 \sigma_{u}\right| 2 \pi_{u}^{2}\left|2 \sigma_{g}\right| 2 \pi_{g}^{2}$ for the seven lowest spin-degenerate orbitals. The highest (eighth) occupied molecular orbital does not fit into this category (i.e., its character is not $2 \sigma_{u}$ ) due to the perturbing symmetry of the $\mathrm{Al}_{2} \mathrm{Li}_{10}$ cluster. The above findings are corroborated in Fig. 3, where the energy levels of $\mathrm{AlLi}_{5}$ correlate well with the corresponding (split) levels of $\mathrm{Al}_{2} \mathrm{Li}_{10}$.

Figure 1 shows that the idea of forming clusters from $\mathrm{AlLi}_{5}$ units does not hold for larger $\mathrm{Al}_{N} \mathrm{Li}_{5 N}$ clusters. This is further demonstrated in Fig. 3, which shows that each cluster size displays its own electronic character. Nevertheless, we see a common behavior for all these clusters: the clear energy separation between the $s$ and $p$ electrons, which evolves from $3.69 \mathrm{eV}$ in $\mathrm{AlLi}_{5}$ to $0.76 \mathrm{eV}$ in $\mathrm{Al}_{10} \mathrm{Li}_{50}$. In order to study this phenomenon more extensively we have performed a spherical harmonics analysis of the KS orbitals in the vicinity of each $\mathrm{Al}$ ion, ${ }^{30}$ and the $s$ and $p$ components obtained for $\mathrm{Al}_{6} \mathrm{Li}_{30}$ and $\mathrm{Al}_{10} \mathrm{Li}_{50}$ are shown in Fig. 4. Each component is averaged over all the $\mathrm{Al}$ ions using the magnitude of the particular orbital in the particular location (Al ion) as a weighting factor. From Fig. 4 we see that the $N$ lowest spindegenerate orbitals of $\mathrm{Al}_{N} \mathrm{Li}_{5 N}$ clusters have a clear $s$ character $\left(\bar{w}_{s} \geqslant 0.80\right)$, whereas the higher orbitals (with a few exceptions) show a clear $p$ character $\left(\bar{w}_{p}=0.47-0.96\right)$ up to

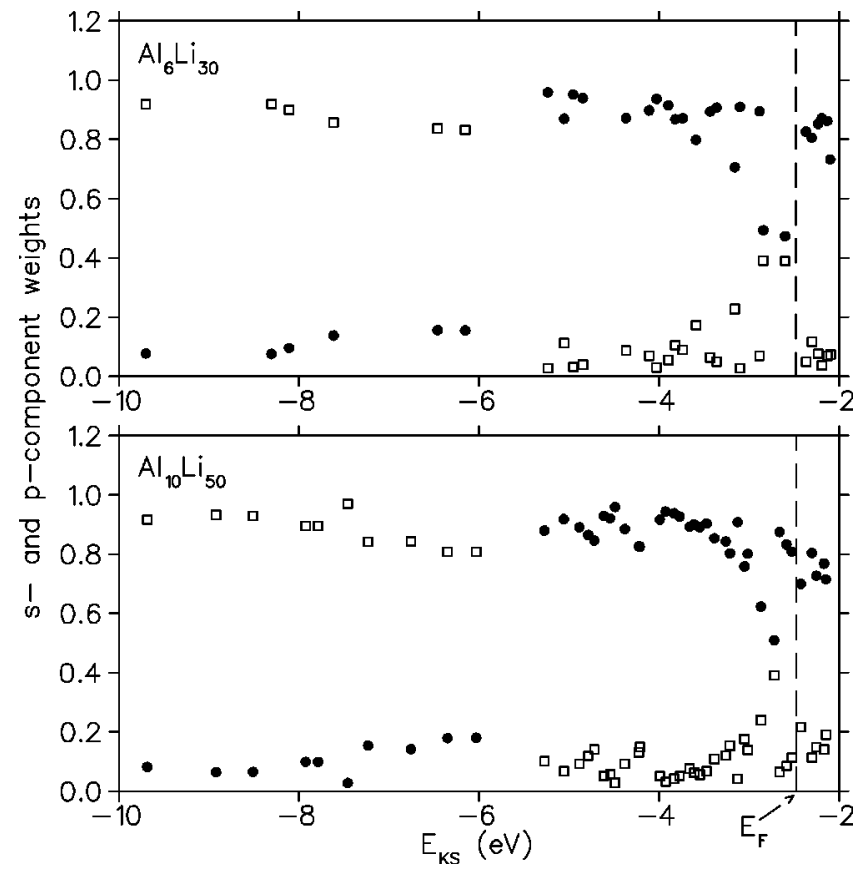

FIG. 4. Weighted $s$ and $p$ components of the KS orbitals of $\mathrm{Al}_{6} \mathrm{Li}_{30}$ and $\mathrm{Al}_{10} \mathrm{Li}_{50}$ clusters. Open squares correspond to $s$ components and solid circles to $p$ components.

the Fermi energy. Similar observations apply to the other $\mathrm{Al}_{N} \mathrm{Li}_{5 N}$ clusters, and we conclude that the $s-p$ hybridization does not occur for the cluster sizes studied here. We expect that this behavior will continue up to relatively large cluster sizes, since even the lithium-rich bulk alloys exhibit identifiable $s$ and $p$ bands. $^{21}$

The charge density and accumulation-depletion isosurfaces of $\mathrm{Al}_{3} \mathrm{Li}_{15}$ are shown in Fig. 5. The amount of total charge inside the two charge density plots is $38 \%$ and $75 \%$, respectively; i.e., in the first case the enclosed charge is equal to the initial valence electron contribution of $\mathrm{Al}$ atoms. The binary nature of $\mathrm{Al}_{3} \mathrm{Li}_{15}$ is evident: The largest charge densities are observed in the vicinity of $\mathrm{Al}$ ions, whereas the rest of the charge is delocalized over the Li region in accordance with the prolate geometry of the cluster. This can also be seen in the accumulation-depletion plot, which shows a complicated charge transfer pattern. As expected, Li ions donate charge to $\mathrm{Al}$ and especially to the interstitial region between $\mathrm{Al}$ and $\mathrm{Li}$ ions, leading to the ionic character of $\mathrm{Al}-\mathrm{Li}$ bonding. However, Al ions also move charge from the center and the apices of the Al triangle, and the largest depletion values are found in these regions. The Al-Al bonds do not participate in the charge transfer process, and the covalent character of these bonds is enhanced. We note that some charge has accumulated at the ends of the prolate cluster, indicating a self-deformation of electron density that might be related to the similar phenomena observed in the jellium model. ${ }^{2,3}$

\section{B. $B 2$ and $B 32$ AlLi cluster alloys}

Cluster geometries studied for $\mathrm{Al}_{32} \mathrm{Li}_{27}$ (B2), $\mathrm{Al}_{30} \mathrm{Li}_{29}$ (B32), $\mathrm{Al}_{64} \mathrm{Li}_{81}$ (B2), and $\mathrm{Al}_{70} \mathrm{Li}_{75}$ (B32) are shown in Fig. 6. All these clusters were initially constructed from the bcc 

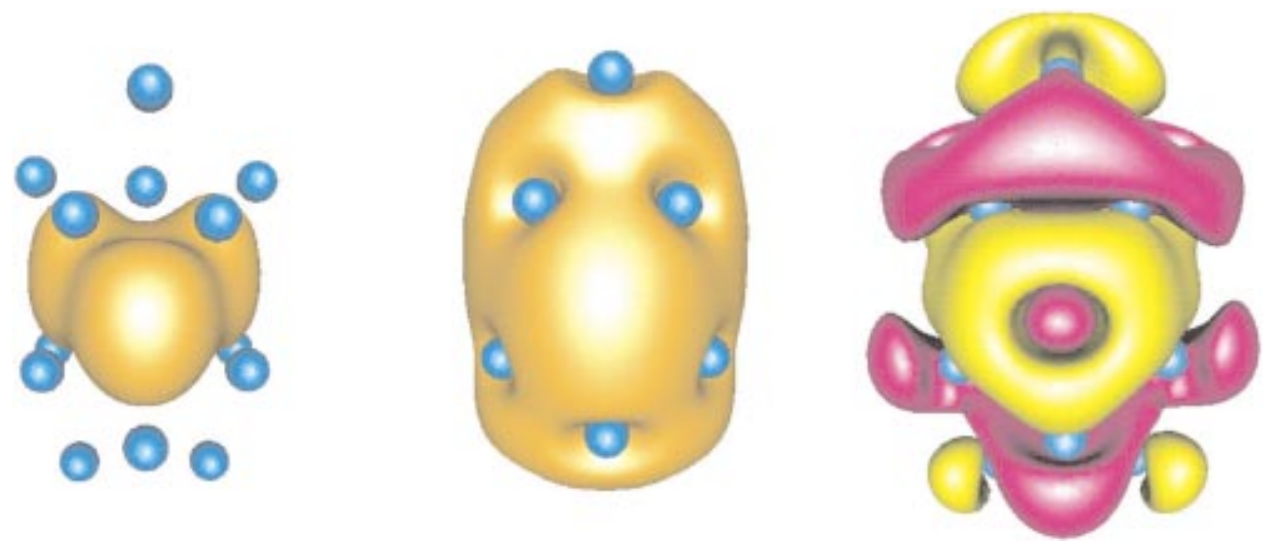

FIG. 5. (Color) Charge density and accumulation-depletion isosurfaces of $\mathrm{Al}_{3} \mathrm{Li}_{15}$ cluster. The density values on the isosurfaces are 0.015 a.u. and 0.005 a.u. corresponding to $38 \%$ and $75 \%$ enclosure of the total charge. The accumulation (yellow) and depletion (violet) values on the isosurfaces are both 0.0005 a.u. (8.3\% and $5.6 \%$ of the maximum values). lattice, where $\mathrm{Al}$ and $\mathrm{Li}$ atom occupations were decided according to the AlLi alloy structure desired (B2 or B32). In order to make the clusters more spherical and to reduce the area of (100) facets, we have chosen a truncated rhombic dodecahedron (59 and 145 atoms) as the underlying cluster geometry. The starting configurations were freely optimized to their corresponding metastable minima with both the local density approximation (LDA) and GGA (we use spindegenerate orbitals here). During the optimization the corners and edges of $\mathrm{Al}_{32} \mathrm{Li}_{27}$ became less pronounced, resulting a nearly spherical shape. The outermost $\mathrm{Al}$ ions gained $\mathrm{Al}$ contacts (only Al-Li nearest-neighbor bonds exist in the pure $B 2$ phase), and the relatively large HOMO-LUMO gap of $\mathrm{Al}_{32} \mathrm{Li}_{27}\left(E_{g}=0.49 \mathrm{eV}\right.$; see Fig. 7) suggests that this structure might be a low-energy isomer. On the other hand, $\mathrm{Al}_{30} \mathrm{Li}_{29}$ maintained its geometry quite well; the most visible changes are observed in the (100) facets, where the tetrahedral coordination of the cluster (B32) induces some twisting.
$\mathrm{Al}_{64} \mathrm{Li}_{81}$ does not show similar rounding to $\mathrm{Al}_{32} \mathrm{Li}_{27}$, the $\mathrm{Al}$ and $\mathrm{Li}$ layers being ordered differently. Together with the increased size this explains the absence of rounding effects. On the other hand, $\mathrm{Al}_{70} \mathrm{Li}_{75}$ exhibits quite similar behavior to $\mathrm{Al}_{30} \mathrm{Li}_{29}$ with its distorted (100) facets. We note that the large (100) facets of $\mathrm{Al}_{70} \mathrm{Li}_{75}$ make the cluster unstable in the GGA and cause it to optimize away from the desired $B 32$ structure. The results reported for $\mathrm{Al}_{70} \mathrm{Li}_{75}$ are for the LDA alone.

The KS energy eigenvalues of $B 2$ and $B 32$ AlLi clusters calculated with GGA are shown in Fig. 7. There is no separation of $s$ and $p$ bands, as in the case of bulk calculations. ${ }^{21}$ Furthermore, pronounced deviations in the overall locations of the eigenvalue spectra indicate differences in the electronic structures of $B 2$ and $B 32$ clusters. According to Guo et $a .^{21}$ the two phases of bulk AlLi alloys have distinctive electronic natures: $B 2$ is more metallic due to the absence of $\mathrm{Al}-\mathrm{Al}$ bonds, whereas $B 32$ resembles more the covalent bonding in bulk Si (diamond lattice). Inspection of charge

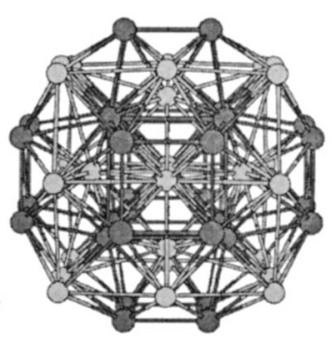

B2

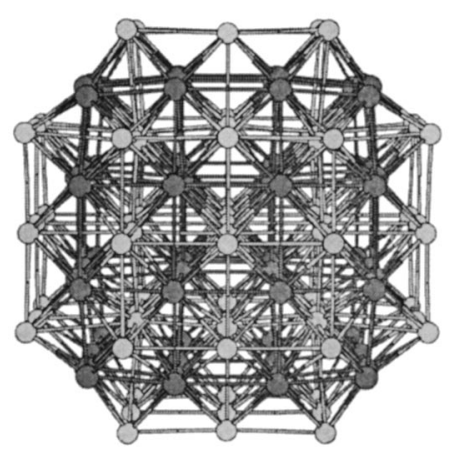

B2

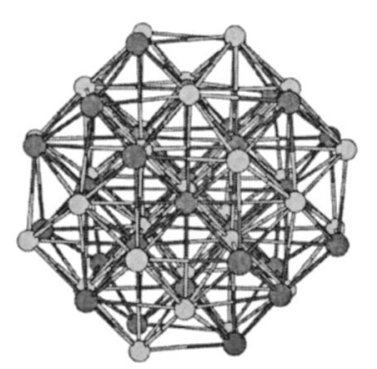

B32

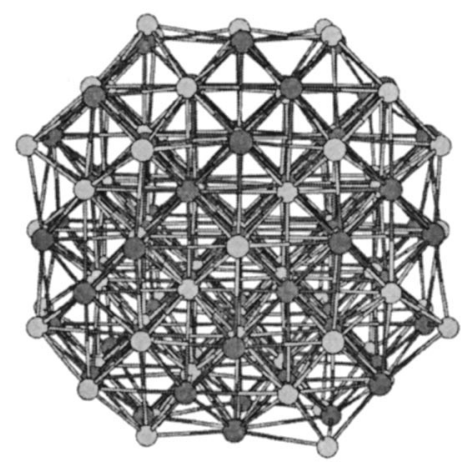

B32
FIG. 6. Optimized geometries of $\mathrm{Al}_{32} \mathrm{Li}_{27}$ (B2), $\quad \mathrm{Al}_{30} \mathrm{Li}_{29}$ (B32), $\quad \mathrm{Al}_{64} \mathrm{Li}_{81}$ (B2), and $\mathrm{Al}_{70} \mathrm{Li}_{75}$ (B32) clusters. $\mathrm{Al}$ atoms are black; Li atoms are gray. 


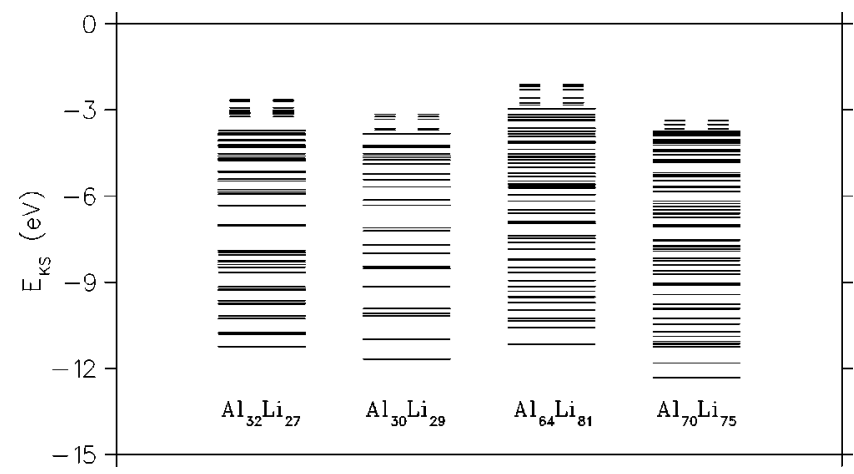

FIG. 7. KS-GGA energy levels of $\mathrm{Al}_{32} \mathrm{Li}_{27}$ (B2), $\mathrm{Al}_{30} \mathrm{Li}_{29}$ (B32), $\mathrm{Al}_{64} \mathrm{Li}_{81}$ (B2), and $\mathrm{Al}_{70} \mathrm{Li}_{75}$ (B32) clusters. The shorter lines correspond to unoccupied orbitals. Note that the orbitals are spin degenerate while the number of electrons is odd for each cluster (the HOMO levels are only partially filled).

density contours (not shown) indicates that this is also the case for the $B 2$ and $B 32$ clusters.

The physical properties of $B 2$ and $B 32$ AlLi clusters are listed in Table II. In addition to the compressibilities $B$ we present the scaled second derivatives $A$ of the total energy, which cause no volume-induced ambiguities [see Eq. (2) below]. The ratio of $\mathrm{Al}$ to $\mathrm{Li}$ ions varies considerably among the cluster sizes studied, and comparisons of average binding energies between different cluster sizes should be made with caution. The relatively high $\bar{E}_{b}$ of $\mathrm{Al}_{32} \mathrm{Li}_{27}$ supports our earlier suggestions of the high stability of this cluster.

In order to calculate the compressibility (bulk modulus)

$$
B=-V\left(\frac{\partial P}{\partial V}\right)_{N}=V\left(\frac{\partial^{2} E}{\partial V^{2}}\right)_{N}
$$

of the clusters, we have chosen any size-related length $l$ (e.g., cluster radius) as the variable, leading to

$$
B=\frac{A}{9 \Omega_{c l}}, \quad A=\frac{1}{N}\left(l^{2} \frac{\partial^{2} E}{\partial l^{2}}\right),
$$

where $\Omega_{c l}=V_{c l} / N$ is the average volume per atom in the cluster, and the scaled second derivative $A$ of the total energy $E$ is calculated using a simple scaling of cluster volume in a region where the harmonic approximation is adequate (for a better method of compressing clusters see, for example, Mar- toňák et $\left.a l . .^{31}\right)$. The quantity $A$ does not depend on the volume. However, in calculating the compressibility $B$ the concept of cluster volume is a major problem that cannot be overcome completely. We integrate spherically over the charge density and deduce the cluster radius and volume $\left(V_{c l}=\frac{4}{3} \pi R_{c l}^{3}\right.$ ) from the radial electron density obtained. ${ }^{32}$ The resulting compressibilities $B$ (see Table II) have considerable uncertainties due to inaccuracies in the volume determination.

The two AlLi phases show different size evolutions (Table II). While the $B 32$ compressibilities exhibit a clear increase from $\mathrm{Al}_{30} \mathrm{Li}_{29}$ to $\mathrm{Al}_{70} \mathrm{Li}_{75}$, it is impossible to assign a similar behavior for $B 2$ clusters. The probable reason is the abovementioned "magic" nature of $\mathrm{Al}_{32} \mathrm{Li}_{27}$ (note also the clear deviation between the LDA and GGA results). Generally, inspection of both scaled second derivatives $A$ and compressibilities $B$ shows that these values are surprisingly close to the corresponding bulk values, although the surface-tovolume ratios of clusters are still considerable. Furthermore, a comparison of calculated $B$ values with the known bulk moduli of pure $\mathrm{Li}$ and Al metals $\left[B_{L i}=11.5 \mathrm{GPa}\right.$ and $B_{A l}$ $=76 \mathrm{GPa}$ (Ref. 27)] indicates that all the clusters are already harder than the pure $\mathrm{Li}$ metal. For the smaller cluster sizes $\mathrm{Al}_{32} \mathrm{Li}_{27}$ and $\mathrm{Al}_{30} \mathrm{Li}_{29}$ we cannot assign any structurerelated differences, but the larger clusters $\mathrm{Al}_{64} \mathrm{Li}_{81}$ and $\mathrm{Al}_{70} \mathrm{Li}_{75}$ show that the latter (B32) is already significantly harder, in agreement with the bulk calculations (see Table II).

\section{CONCLUSION}

We have studied AlLi clusters using the first-principles BO-LSD-MD simulation method in conjunction with the LSD approximation and GGA parametrizations of the exchange-correlation energy, separable nonlocal pseudopotentials, and a plane-wave basis set. In the case of $\mathrm{Al}_{N} \mathrm{Li}_{5 N}$ $(N=1-6,10)$ clusters, the optimal cluster geometries are generated via a simulated annealing strategy, where the dynamical finite-temperature simulations are performed using the BO approximation and the Hellmann-Feynman theorem. The number of different isomer configurations increases in binary clusters even faster than in the homonuclear aggregates, and we cannot guarantee that all the structures obtained are indeed the lowest-energy isomers of the corresponding cluster sizes. However, we believe that the major structural trends of Li-rich AlLi clusters are well described.

TABLE II. Properties of $B 2$ and $B 32$ AlLi cluster alloys: number of valence electrons, average binding energy (LDA/GGA), scaled second derivative of total energy (LDA/GGA), determined cluster radius, and compressibility (bulk modulus). Results from the LDA bulk calculations of Ref. 21 are also presented.

\begin{tabular}{lccccc}
\hline \hline Cluster & $N_{e l}$ & $\bar{E}_{b}$ (eV/atom) & $A(\mathrm{eV})$ & $R_{c l}\left(a_{0}\right)$ & $B(\mathrm{GPa})$ \\
\hline $\mathrm{Al}_{32} \mathrm{Li}_{27}(B 2)$ & 123 & $2.64 / 2.32$ & $31.8 / 41.4$ & $12.5 \pm 1$ & $21-45$ \\
$\mathrm{Al}_{30} \mathrm{Li}_{29}(B 32)$ & 119 & $2.47 / 2.17$ & $35.7 / 36.0$ & $13 \pm 1$ & $22-35$ \\
$\mathrm{Al}_{64} \mathrm{Li}_{81}(B 2)$ & 273 & $2.63 / 2.30$ & $31.5 / 32.3$ & $16 \pm 1$ & $27-41$ \\
$\mathrm{Al}_{70} \mathrm{Li}_{75}(B 32)$ & 285 & $2.64 />2.33$ & $48.5 / \ldots$ & $16 \pm 1$ & $41-59$ \\
\hline Bulk $(B 2)$ & $\ldots$ & 3.30 & 34.8 & $\ldots$ & 42 \\
Bulk $(B 32)$ & $\ldots$ & 3.39 & 49.8 & $\ldots$ & 58 \\
\hline \hline
\end{tabular}


We have observed structural changes in the geometries of lithium-rich $\mathrm{Al}_{N} \mathrm{Li}_{5 N}$ cluster alloys. $\mathrm{Al}-\mathrm{Al}$ bonds start to break as the cluster size is increased $(N \geqslant 5)$, leading to a mixture of $\mathrm{Al}$ and $\mathrm{Li}$ ions, where the resulting $\mathrm{Al}$ skeleton finally forms a chainlike configuration $(N=10)$. We conclude that it is not possible to build larger lithium-rich AlLi clusters where the $\mathrm{Al}$ ions comprise a compact core (e.g., icosahedral $\left.\mathrm{Al}_{13} \mathrm{Li}_{42}\right)$. Furthermore, the idea of building stable $\mathrm{Al}_{N} \mathrm{Li}_{5 N}$ clusters from "magic" $\mathrm{AlLi}_{5}$ units does not apply after $\mathrm{Al}_{2} \mathrm{Li}_{10}$. This is further supported by our calculations - that the total energies of the larger clusterassembled metastable isomers are much higher than for the corresponding lowest-energy isomer (for example, assembled $\mathrm{Al}_{5} \mathrm{Li}_{25}$ isomer is $0.145 \mathrm{eV} /$ atom higher in energy). We note that there are other metallic compounds, such as $\mathrm{Na}_{4} \mathrm{~Pb}_{4},{ }^{33}$ where the cluster-assembling idea is fulfilled.

The electronic structures of $\mathrm{Al}_{N} \mathrm{Li}_{5 N}$ clusters show that the $s-p$ hybridization does not arise at small cluster sizes, and that there is always a clear gap between the $s$ and $p$ bands. This result reflects a significantly different behavior from pure Al clusters, where the hybridization effects can be observed quite early $(N \approx 4) .{ }^{14}$ Consequently, we predict that for the corresponding cluster anions there should be a clear band gap in the photoelectron spectrum about $2-3 \mathrm{eV}$ above the threshold. Using $\mathrm{Al}_{3} \mathrm{Li}_{15}$ as an example, we have demonstrated an interesting charge transfer phenomenon, where ionic $\mathrm{Al}-\mathrm{Li}$ and covalent $\mathrm{Al}-\mathrm{Al}$ bonds are formed.

In order to study the evolution of certain bulk properties, we have considered two different cluster sizes ( $N_{\text {atoms }}$ $=59,145)$ and two different phases of bulk AlLi alloy $(B 2$ and $B 32$ ) in the same external confinement (truncated rhombic dodecahedron). Although we do not expect that any of the isomers studied is the most stable isomer of the corresponding cluster size, we observe that $\mathrm{Al}_{32} \mathrm{Li}_{27}$ has properties typical of particularly stable metal clusters. Already in this size regime we observe that the electronic structures of different bulk phases have their own characteristic properties. $B 2$ is more metallic due to missing Al nearest neighbors, whereas $B 32$ has a strong covalent nature because of its tetrahedrally coordinated (diamond) sublattice of Al ions. Similar observations can also be made with respect to the compressibilities of $\mathrm{Al}_{64} \mathrm{Li}_{81}$ (B2) and $\mathrm{Al}_{70} \mathrm{Li}_{75}$ (B32) clusters, where the covalent $\mathrm{Al}-\mathrm{Al}$ bonds make $B 32$ significantly harder.

\section{ACKNOWLEDGMENTS}

Computations were performed on a Cray T3E at the Center for Scientific Computing (CSC), Espoo, Finland. We thank U. Landman and H. Häkkinen for several valuable discussions, and R. O. Jones for a critical reading of the manuscript. This work has been supported by the Academy of Finland under the Finnish Center of Excellence Program 2000-2005 (Project No. 44875, Nuclear and Condensed Matter Program at JYFL). J.A. acknowledges a grant from the Väisälä Foundation.
*Present address: Institut für Festkörperforschung, Forschungszentrum Jülich, D-52425 Jülich, Germany.

${ }^{1}$ W. D. Knight, K. Clemenger, W. A. de Heer, W. A. Saunders, M. Y. Chou, and M. L. Cohen, Phys. Rev. Lett. 52, 2141 (1984); 53, 510(E) (1984).

${ }^{2}$ M. Brack, Rev. Mod. Phys. 65, 676 (1993).

${ }^{3}$ C. Yannouleas and U. Landman, in Large Clusters of Atoms and Molecules, edited by T. P. Martin (Kluwer, Dordrecht, 1996), p. 131.

${ }^{4}$ W. A. de Heer, Rev. Mod. Phys. 65, 611 (1993).

${ }^{5}$ W. D. Knight, W. A. de Heer, K. Clemenger, and W. A. Saunders, Solid State Commun. 53, 445 (1985); W. D. Knight, W. A. de Heer, W. A. Saunders, K. Clemenger, M. Y. Chou, and M. L. Cohen, Chem. Phys. Lett. 134, 1 (1987).

${ }^{6}$ M. M. Kappes, P. Radi, M. Schär, and E. Schumacher, Chem. Phys. Lett. 119, 11 (1985).

${ }^{7}$ M. Heinebrodt, N. Malinowski, F. Tast, W. Branz, I. M. L. Billas, and T. P. Martin, J. Chem. Phys. 110, 9915 (1999).

${ }^{8}$ I. Boustani, W. Pewestorf, P. Fantucci, V. Bonačić-Koutecký, and J. Koutecký, Phys. Rev. B 35, 9437 (1987).

${ }^{9}$ O. Sugino and H. Kamimura, Phys. Rev. Lett. 65, 2696 (1990).

${ }^{10}$ V. Bonačić-Koutecký, P. Fantucci, and J. Koutecký, Chem. Rev. 91, 1035 (1991).

${ }^{11}$ M.-W. Sung, R. Kawai, and J. H. Weare, Phys. Rev. Lett. 73, 3552 (1994).

${ }^{12}$ R. O. Jones, J. Chem. Phys. 99, 1194 (1993).

${ }^{13}$ S. H. Yang, D. A. Drabold, J. B. Adams, and A. Sachdev, Phys. Rev. B 47, 1567 (1993).

${ }^{14}$ J. Akola, H. Häkkinen, and M. Manninen, Phys. Rev. B 58, 3601
(1998); J. Akola, M. Manninen, H. Häkkinen, U. Landman, X. Li, and L.-S. Wang, ibid. 60, 11297 (1999); 62, 13216 (2000).

${ }^{15}$ K. E. Schriver, J. L. Persson, E. C. Honea, and R. L. Whetten, Phys. Rev. Lett. 64, 2539 (1990).

${ }^{16}$ H.-P. Cheng, R. N. Barnett, and U. Landman, Phys. Rev. B 48, 1820 (1993).

${ }^{17}$ V. Shah and D. G. Kanhere, J. Phys.: Condens. Matter 8, L253 (1996); V. Shah, D. G. Kanhere, C. Majumder, and P. G. Das, ibid. 9, 2165 (1997).

${ }^{18}$ A. M. Vichare and D. G. Kanhere, Eur. Phys. J. D 4, 89 (1998).

${ }^{19}$ V. Kumar, Phys. Rev. B 60, 2916 (1999).

${ }^{20}$ A. L. McAlister, Bulk Alloy Phase Diagrams 3, 177 (1982).

${ }^{21}$ X.-Q. Guo, R. Podloucky, and A. J. Freeman, Phys. Rev. B 40, 2793 (1989); 42, 10912 (1990).

${ }^{22}$ R. N. Barnett and U. Landman, Phys. Rev. B 48, 2081 (1993).

${ }^{23}$ N. Troullier and J. L. Martins, Phys. Rev. B 43, 1993 (1991). For the lithium $2 s$ and aluminum $3 s^{2} 3 p^{1}$ valence electrons, we use $s$-nonlocal and $p$-local components with cutoff radii of 2.2 and $2.8(\mathrm{Li})$ and 2.1 and $2.5 a_{0}(\mathrm{Al})$, respectively. The KS orbitals are expanded in a plane-wave basis with a cutoff of $15.4 \mathrm{Ry}$. The resulting dissociation energies and dimer bond lengths are 1.79 $\mathrm{eV}$ and $2.52 \AA$ for $\mathrm{Al}_{2}, 0.78 \mathrm{eV}$ and $2.69 \AA$ for $\mathrm{Li}_{2}$, and $1.00 \mathrm{eV}$ and $2.88 \AA$ for AlLi.

${ }^{24}$ S. H. Vosko, L. Wilk, and M. Nusair, Can. J. Phys. 58, 1200 (1980); S. H. Vosko and L. Wilk, J. Phys. C 15, 2139 (1982).

${ }^{25}$ J. P. Perdew, K. Burke, and M. Ernzerhof, Phys. Rev. Lett. 77, 3865 (1996); 78, 1396(E) (1997).

${ }^{26}$ C. Kittel, Introduction to Solid State Physics, 7 th ed. (Wiley, New York, 1996). 
${ }^{27}$ N. W. Ashcroft and N. D. Mermin, Solid State Physics, international ed. (Saunders College Publishing, New York, 1976).

${ }^{28}$ R. O. Jones and O. Gunnarsson, Rev. Mod. Phys. 61, 689 (1989).

${ }^{29}$ The KS orbitals of $\mathrm{Al}_{5} \mathrm{Li}_{25}$ are projected onto spherical harmonic components, where the origin is placed at the center of charge. Inspection of the obtained angular moment component weights reveals that the KS orbitals do not fit into the SJM type of sequence $1 s^{2}\left|1 p^{6}\right| 1 d^{10}\left|2 s^{2}\right| 1 f^{14} \mid 2 p^{6}$ for 40 electrons.

${ }^{30}$ In order to study $s-p$ hybridization effects a spherical harmonics analysis of $\mathrm{Al}_{N} \mathrm{Li}_{5 N}(N \geqslant 2)$ clusters is performed in Al-centered finite spheres which roughly touch each other. For $\mathrm{Al}_{2} \mathrm{Li}_{10}$ the sphere radius is $3.0 a_{0}$ and for the other cluster sizes it is $2.6 a_{0}$. Also the weights of KS orbitals inside each sphere are calculated during this analysis.

${ }^{31}$ R. Martoňák, C. Molteni, and M. Parrinello, Phys. Rev. Lett. 84, 682 (2000).

${ }^{32}$ For the cluster radius $R_{c l}$ it is required that $95 \%$ of the total charge of the cluster is inside a corresponding sphere. In addition, an error of $\pm 1 a_{0}$ is included causing the enclosed charge to vary in the range of $90 \%-98 \%$.

${ }^{33}$ L. M. Molina, J. A. Alonso, and M. J. Stott, J. Chem. Phys. 111, 7053 (1999). 